\title{
Reduction of non-variational bi-Hamiltonian system of shallow-water waves propagation via symmetry approach
}

\author{
Adil Jhangeer $\mathrm{a}^{\text {* }}$ \\ ${ }^{a}$ Department of Electrical Engineering, Namal College, Talagang Road, Mianwali 42250, Pakistan \\ *Corresponding author E-mail: adil.jhangeer@gmail.com
}

\section{Article Info}

Keywords: Lagrangian, Noether operator, Conserved quantities

2010 AMS: $35 Q X X$

Received: 31 March 2018

Accepted: 10 May 2018

Available online: 27 May 2018

\begin{abstract}
In this paper, non-variational bi-Hamiltonian system of shallow-water waves propagation is considered. Lie point generators are calculated and one dimensional optimal system of its subalgebras up to conjugacy classes are reported. Then similarity variables are computed by using these conjugacy classes which are further utilized for the reduction of considered system. Then, a transformation is used to convert the system from non-variational to variational system, thus standard Lagrangian is computed. Noether operators are calculated by using Noether approach and local conserved quantity is discussed for the new fourth order system of partial differential equations (PDEs). Further, inverse transformation is applied to get the corresponding local conserved quantity for the considered non-variational problem. Moreover, this local conservation law with the help of double reduction theorem is utilized to reduce the system.
\end{abstract}

\section{Introduction}

Exact solutions of a system of nonlinear PDEs have lot of importance in the solution of initial or boundary value problems. It also helps to see the exact inner picture of the phenomena. For higher order nonlinear system of PDEs, one does not has the enough methods to find the exact solutions. Symmetry approach is one of the powerful tools for analyzing such systems. Lie group theory is also one of the most interesting and active field of research for the solution of nonlinear PDEs. It is a systematic technique to construct a class of exact solutions or reduce the considered differential equation into one of its simplest form. It also provides applicable approach to find the closed form solution of differential equation. There are lot of efficient techniques available for the computation of exact solutions of differential equations. Some well known techniques are separation of variables, traveling wave solution, self-similar solutions, solution by using ansatz and exponential self-similar solutions. These approaches are the particular case of Lie group method (see [15]).

Reduction of a differential equation by using Noether approach is one of the aspect of Lie theory. Conserved quantities play a vital role in the theory of differential equations. Solution and reduction of PDEs are some of the aspects where conserved quantities have its significant importance. On the derivation of conservation laws active research efforts have been made in the last few decades. One of the elegant and systematic approach is developed by Noether [12]. Direct method [2], characteristics approach [20] and the partial Noether approach [10] are the approaches which have been used frequently in literature. Computing utilities for the calculation of conservation laws are also practised in past (see $[4,6,7,8,9,21,22])$.

A first systematic method for finding the conservation laws [12] of the variational problems is given by Emmy Noether. She found a link between the symmetries and conservation laws [12]. She concluded that each symmetry corresponds to a conserved quantity. This approach is valid for the variational problems and thus depends on the existence of standard Lagrangian. There is a big class of PDEs and system of PDEs which do not posses standard Lagrangian, thus Noether approach is not applicable. In this article, we will apply Noether approach to the non-variational system of PDEs. For this we will use the following steps:

(i): Convert the non-variational system of PDEs into variational problem by using suitable transformation.

(ii): Standard Lagrangian is calculated and Noether approach is applied in new coordinates. 
(iii): Noether operators and corresponding conservation laws are computed.

(iv): Use an inverse transformation to convert the conserved quantities into the coordinates of original problem.

The bi-Hamiltonian Boussinesq system [1]:

$$
u_{t}-\Omega v_{x}=0, \quad v_{t}-\Omega\left(u_{x x x}+8 u u_{x}\right)=0,
$$

describes shallow-water wave propagation in both directions, where $\Omega$ is a real constant.

In the past, lot of work has been done on the different forms of shallow-water waves propagation by means of Lie symmetry analysis. In [14], M. Pandey discussed the one dimensional shallow water equation by using Lie approach. Exact solutions of one dimensional axis symmetric flow of shallow water equations involving bores was computed with the help of point symmetries in [17]. Plebanski's second heavenly equation and 2+1-dimensional hamiltonian integrable system and is discussed in [11,23] by means of symmetries. The group analysis and an infinite aggregate of non-degenerate solutions of the equations of one-dimensional model of shallow water with a straight bottom is discussed in [5].

The pattern of this paper is as follow. In Section 2, basic operators are discussed. The Lie point generators and reduction by using similarity variables for the system (1.1) are discussed in Section 3. Local conserved quantity and double reduction for the considered system is computed in Section 4. At last conclusion is provided.

\section{Basic operators}

Let $(t, x)$ be independent variables and $(u, v)$ be dependent variables. Consider the third order system of PDEs with two independent and dependent variables i.e.

$$
E_{1}\left(t, x, u, v, u_{t}, v_{t}, \ldots\right)=0, \quad E_{2}\left(t, x, u, v, u_{t}, v_{t}, \ldots\right)=0
$$

(1): A vector field:

$$
Y=\tau(t, x, u, v) \frac{\partial}{\partial t}+\xi(t, x, u, v) \frac{\partial}{\partial x}+\phi(t, x, u, v) \frac{\partial}{\partial u}+\eta(t, x, u, v) \frac{\partial}{\partial v}
$$

is a Lie point symmetry generator of (2.1) if it satisfies the invariance condition i.e.

$$
Y^{[3]} E_{1}=0, \quad Y^{[3]} E_{2}=0, \quad \text { whenever } \quad E_{1}=0, \quad E_{2}=0,
$$

where $Y^{[3]}$ is the third prolongation [13].

(2): If $u \rightarrow U_{x}$ and $v \rightarrow V_{x}$ in (2.1) then the given third order system becomes fourth order system in $U, V$ variables, i.e.

$$
G_{1}\left(t, x, U, V, U_{t}, V_{t}, \ldots\right)=0, \quad G_{2}\left(t, x, U, V, U_{t}, V_{t}, \ldots\right)=0 .
$$

(3): The Euler operator is:

$$
\begin{gathered}
\frac{\delta}{\delta U}=\frac{\partial}{\partial U}-D_{t} \frac{\partial}{\partial U_{t}}-D_{x} \frac{\partial}{\partial U_{x}}+D_{t}^{2} \frac{\partial}{\partial U_{t t}}+D_{x}^{2} \frac{\partial}{\partial U_{x x}}+\ldots \\
\frac{\delta}{\delta V}=\frac{\partial}{\partial V}-D_{t} \frac{\partial}{\partial V_{t}}-D_{x} \frac{\partial}{\partial V_{x}}+D_{t}^{2} \frac{\partial}{\partial V_{t t}}+D_{x}^{2} \frac{\partial}{\partial V_{x x}}+\ldots
\end{gathered}
$$

where

$$
D_{t}=\frac{\partial}{\partial t}+U_{t} \frac{\partial}{\partial U}+V_{t} \frac{\partial}{\partial V}+U_{t t} \frac{\partial}{\partial U_{t}}+V_{t t} \frac{\partial}{\partial V_{t}}+U_{t x} \frac{\partial}{\partial U_{x}}+\ldots
$$

and

$$
D_{x}=\frac{\partial}{\partial x}+U_{x} \frac{\partial}{\partial U}+V_{x} \frac{\partial}{\partial V}+U_{x x} \frac{\partial}{\partial U_{x}}+V_{x x} \frac{\partial}{\partial V_{x}}+U_{t x} \frac{\partial}{\partial U_{t}}+\ldots
$$

are known as the total derivative operators.

(4): The generalized operator is defined by

$$
X=\tau \frac{\partial}{\partial t}+\xi \frac{\partial}{\partial x}+\phi \frac{\partial}{\partial U}+\eta \frac{\partial}{\partial V}+\phi^{x} \frac{\partial}{\partial U_{x}}+\eta^{x} \frac{\partial}{\partial V_{x}}+\phi^{t} \frac{\partial}{\partial U_{t}}+\ldots
$$

(5): A standard Lagrangian $L=L\left(t, x, U, V, U_{t}, V_{t} \ldots\right) \in A$ (space of differential functions) and satisfies:

$$
\frac{\delta L}{\delta U}=0 \text { and } \frac{\delta L}{\delta V}=0 .
$$

(6): The generalized operator is known as Noether operator associated with a standard Lagrangian $L$ if it satisfies:

$$
X^{[2]} L+L\left(\tau_{t}+\tau_{U} U_{t}+\tau_{V} V_{t}+\xi_{x}+\xi_{U} U_{x}+\xi_{V} V_{x}\right)=B_{t}^{1}+B_{U}^{1} U_{t}+B_{V}^{1} V_{t}+B_{x}^{2}+B_{U}^{2} U_{x}+B_{V}^{2} V_{x} .
$$


In Eq.(2.9), $B^{i}$,s are known as the gauge terms, while $X^{[2]}$ is the second prolongation of the generator $X$.

(7): The equation:

$$
\left(T^{1}\right)_{t}+\left(T^{2}\right)_{x}=0
$$

evaluated on the solution space given by (2.1) is known as the conservation laws for Eq. (2.1). The vector $T=\left(T^{1}, T^{2}\right)$ is a conserved vector where $T^{1}, T^{2}$ are its components.

The conserved vectors of the system (2.1) associated with a Noether operator $X$ can be determined from the formula:

$$
T^{i}=B^{i}-N^{i}(L) \quad i=1,2,
$$

where

$$
\begin{aligned}
N^{1}= & \tau+\left(\phi-\tau U_{t}-\xi U_{x}\right) \frac{\delta}{\delta U_{t}}+\left(\eta-\tau V_{t}-\xi V_{x}\right) \frac{\delta}{\delta V_{t}}-D_{t}\left(\phi-\tau U_{t}-\xi U_{x}\right) \frac{\delta}{\delta U_{t t}} \\
& -D_{t}\left(\eta-\tau V_{t}-\xi V_{x}\right) \frac{\delta}{\delta V_{t t}}-D_{x}\left(\phi-\tau U_{t}-\xi U_{x}\right) \frac{\delta}{\delta U_{t x}}+\ldots, \\
N^{2}= & \xi+\left(\phi-\tau U_{t}-\xi U_{x}\right) \frac{\delta}{\delta U_{x}}+\left(\eta-\tau V_{t}-\xi V_{x}\right) \frac{\delta}{\delta V_{x}}-D_{x}\left(\phi-\tau U_{t}-\xi U_{x}\right) \frac{\delta}{\delta U_{x x}} \\
& -D_{x}\left(\eta-\tau V_{t}-\xi V_{x}\right) \frac{\delta}{\delta V_{x x}}-D_{t}\left(\phi-\tau U_{t}-\xi U_{x}\right) \frac{\delta}{\delta U_{t x}}+\ldots .
\end{aligned}
$$

Following statements are taken from [3, 18, 19].

(8): For nonlocal variable $\omega$ such that $T^{1}=\omega_{x}$ and $T^{2}=-\omega_{t}$. While using similarity variables one can have $T^{r}=\omega_{s}$ and $T^{s}=-\omega_{r}$, where

$$
T^{r}=\frac{T^{1} D_{t}(r)+T^{2} D_{x}(r)}{D_{t}(r) D_{x}(s)-D_{x}(r) D_{t}(s)}, \quad T^{s}=\frac{T^{1} D_{t}(s)+T^{2} D_{x}(s)}{D_{t}(r) D_{x}(s)-D_{x}(r) D_{t}(s)},
$$

so that $D_{r} T^{r}+D_{s} T^{s}=0$.

Theorem 2.1. If system (2.1) admits a nontrivial conserved form that has at least one associated symmetry in every reduction can be reduced to a non linear system of $2^{\text {nd }}$ order ordinary differential equations namely $T^{r}=K$, while $T^{r}$ is defined in (2.14).

\section{Lie Point Symmetries}

In this section, we will apply standard method for finding infinitesimal generators for (1.1). Using (1.1) in (2.2) one can have an overdetermined system of linear PDEs that corresponds the following Lie point symmetries [13]:

$$
Y_{1}=t \frac{\partial}{\partial t}+\frac{x}{2} \frac{\partial}{\partial x}-u \frac{\partial}{\partial u}-\frac{3 v}{2} \frac{\partial}{\partial v}, Y_{2}=\frac{\partial}{\partial t}, \quad Y_{3}=\frac{\partial}{\partial x}, \quad Y_{4}=\frac{\partial}{\partial v}
$$

After rescalling

$$
\mathbf{Y}_{\mathbf{1}}=\frac{2}{3} Y_{1}, \mathbf{Y}_{\mathbf{2}}=Y_{2}, \mathbf{Y}_{\mathbf{3}}=Y_{3} \text { and } \mathbf{Y}_{\mathbf{4}}=Y_{4}
$$

By the definition of a subalgebra, one can easily conclude that there are infinite number of a one-dimensional subalgebras of

$$
L=\left\{\mathbf{Y}_{1}, \mathbf{Y}_{\mathbf{2}}, \mathbf{Y}_{\mathbf{3}}, \mathbf{Y}_{\mathbf{4}}\right\} .
$$

The greatest or best representative of these algebras is called optimal system of one-dimensional subalgebra. In view of all these we can write an arbitrary element from $L$ i.e.

$$
\mathbf{Y}=a \mathbf{Y}_{\mathbf{1}}+b \mathbf{Y}_{\mathbf{2}}+c \mathbf{Y}_{\mathbf{3}}+d \mathbf{Y}_{\mathbf{4}}
$$

where $a, b, c$ and $d$ are real constants. Before going to write down optimal system for the one-dimensional algebra it should be noted that $£_{i}$ and $£_{j}$ are said to be equivalent conjugacy classes if

$$
£_{i}=\operatorname{Ad} Y_{i}\left(£_{j}\right)
$$

where $Y_{i} \in L_{1}$ and

$$
A d\left[\exp \left(t Y_{i}\right)\right] Y_{j}=Y_{j}-t\left[Y_{i}, Y_{j}\right]+\frac{t^{2}}{2}\left[Y_{i},\left[Y_{i}, Y_{j}\right]\right]-\cdots
$$

Now our next task is to simplify (3.2) by applying a carefully chosen adjoint transformation [16] that gives:

$$
\begin{gathered}
£_{1}=<\mathbf{Y}_{\mathbf{1}}>, \quad £_{2}=<\mathbf{Y}_{\mathbf{2}}>, \quad £_{3}=<\mathbf{Y}_{\mathbf{3}}>, \quad £_{4}=<\mathbf{Y}_{\mathbf{4}}>, \\
£_{5}=<\mathbf{Y}_{\mathbf{4}}+\varepsilon \mathbf{Y}_{\mathbf{3}}>, \quad £_{6}=<\mathbf{Y}_{\mathbf{2}}+\varepsilon \mathbf{Y}_{\mathbf{3}}>, £_{7}=<\mathbf{Y}_{\mathbf{4}}+\varepsilon \mathbf{Y}_{\mathbf{2}}+\alpha \mathbf{Y}_{\mathbf{3}}>,
\end{gathered}
$$

where $\varepsilon= \pm 1$ and $\alpha \neq 0$. 


\subsubsection{Reduction by calculating similarity variables}

$<\mathbf{Y}_{1}>$

This operator has the following characteristic system

$$
\frac{d t}{t}=\frac{d x}{\left(\frac{x}{2}\right)}=\frac{d u}{-u}=\frac{d v}{\left(\frac{-3 v}{2}\right)} .
$$

(3.3) can be separated into the following linear equations

$$
\text { (i) : } \frac{d t}{t}=\frac{d x}{\left(\frac{x}{2}\right)}, \quad(i i): \frac{d t}{t}=\frac{d u}{-u}, \quad(i i i): \frac{d x}{\left(\frac{x}{2}\right)}=\frac{d v}{\left(\frac{-3 v}{2}\right)} .
$$

Integrating $(3.4(\mathrm{i})),(3.4(\mathrm{ii}))$ and (3.4 (iii)) one by one we get the following results respectively:

$$
\xi=\frac{t}{x^{2}}, \quad u=\frac{P(\xi)}{t}, \quad v=\frac{Q(\xi)}{x^{3}} .
$$

Substituting the obtained invariants in (1.1) we obtain the following system of ordinary differential equation:

$$
\begin{gathered}
\frac{d P}{d \xi}+2 \Omega \xi^{2} \frac{d Q}{d \xi}-\frac{P}{\xi}+3 \Omega \xi Q=0, \\
8 \Omega \xi^{3} \frac{d^{3} P}{d \xi^{3}}+36 \Omega \xi^{2} \frac{d^{2} P}{d \xi^{2}}+8(3 \xi+2 P) \Omega \frac{d P}{d \xi}-\xi \frac{d Q}{d \xi}=0 .
\end{gathered}
$$

Similarly, working on the same line we discuss each class one by one.

$<\mathbf{Y}_{\mathbf{2}}>$

$$
\xi=x, \quad u=P(\xi), \quad v=Q(\xi),
$$

system after using similarity variables we have:

$$
\frac{d Q}{d \xi}=0, \quad \frac{d^{3} P}{d \xi^{3}}+8 P \frac{d P}{d \xi}=0
$$

$<\mathbf{Y}_{3}>$

For this case, one can have constant solution.

$<\mathbf{Y}_{4}>$

It does not give any new solution.

$<\mathbf{Y}_{\mathbf{4}}+\varepsilon \mathbf{Y}_{\mathbf{3}}>$

$$
\xi=t, \quad u=P(\xi), \quad v=\frac{x}{\varepsilon}+Q(\xi) .
$$

System (1.1) after using similarity variables yields the following solution:

$$
v=\frac{x}{\varepsilon}+c_{1}, \quad u=\frac{\Omega t}{\varepsilon}+c_{2} .
$$

$<\mathbf{Y}_{\mathbf{2}}+\varepsilon \mathbf{Y}_{\mathbf{3}}>$

Similarity variables:

$$
\xi=\varepsilon t-x, \quad u=P(\xi), \text { and } v=Q(\xi)
$$

converts the system (1.1) into:

$$
\frac{d P}{d \xi}+\Omega \frac{d Q}{d \xi}=0, \quad \Omega^{2} \frac{d^{3} P}{d \xi^{3}}+\left(\varepsilon+8 \Omega^{2} P\right) \frac{d P}{d \xi}=0
$$

$<\mathbf{Y}_{\mathbf{4}}+\varepsilon \mathbf{Y}_{\mathbf{2}}+\alpha \mathbf{Y}_{\mathbf{3}}>$

$$
\xi=\varepsilon \alpha t-\varepsilon x, \quad u=P(\xi), \quad v=\frac{t}{\varepsilon}+Q(\xi) .
$$

System after using similarity variables we have:

$$
\alpha \frac{d P}{d \xi}+\Omega \varepsilon \frac{d Q}{d \xi}=0, \quad \Omega \varepsilon^{3} \frac{d^{3} P}{d \xi^{3}}-8 \Omega P \frac{d P}{d \xi}-\alpha \frac{d Q}{d \xi}-\frac{1}{\varepsilon}=0 .
$$

\section{Noether operators and conservation laws}

In this section, we will calculate the conserved quantities of the system of shallow-water waves propagation. 


\subsection{System of shallow-water waves propagation}

It should be noted that system (1.1) belongs to the class of non-variational problems and hence does not possess a standard Lagrangian. In order to make system (1.1) variational let us take $u=U_{x}$ and $v=V_{x}$. The system (1.1) becomes

$$
U_{t x}-\Omega V_{x x}=0, \quad V_{t x}-\Omega\left(U_{x x x x}+8 U_{x} U_{x x}\right)=0 .
$$

The standard Lagrangian for system (4.1) is:

$$
L=\frac{\Omega}{2} U_{x x}^{2}+\frac{\Omega}{2} V_{x}^{2}+\frac{4}{3} \Omega U_{x}^{3}-\frac{1}{2} U_{t} V_{x}-\frac{1}{2} U_{x} V_{t}
$$

Using Eq.(4.2) in Eq.(2.9) and separating with respect to derivatives of $U$ and $V$ one gets:

$$
\begin{gathered}
(i): \xi_{x}=0,(i i): \xi_{t}=0,(i i i): \xi_{U}=0,(i v): \xi_{V}=0,(v): \eta_{U}=0, \\
(i): \tau_{V}=0,(i i): \tau_{U}=0,(i i i): \tau_{x}=0,(i v): \tau_{t}=0,(v): \eta_{V}=0, \\
(i): \phi_{U}=0,(i i): \phi_{V}=0,(i i i): \phi_{x}=0,(i i i): \phi_{t}=0, \\
(i): B_{U}^{1}=-\frac{1}{2} \eta_{x},(i i): B_{V}^{1}=0,(i i i): B_{U}^{2}=-\frac{1}{2} \eta_{t},(i v): B_{V}^{2}=\Omega \eta_{x}
\end{gathered}
$$

and

$$
B_{t}^{1}+B_{x}^{2}=0
$$

The solution of Eqs. (4.3)-(4.7) is

$$
\begin{gathered}
\tau=c_{1}, \quad \xi=c_{2}, \quad \phi=a, \quad \eta=b+c_{3} x \\
B^{1}=-\frac{1}{2} c_{3} U+\lambda(t, x), \quad B^{2}=-\frac{1}{2} b_{t} U-\frac{1}{2} a_{t} V+c_{3} \Omega V+\delta(t, x) .
\end{gathered}
$$

where

$$
\lambda_{t}+\delta_{x}=0,
$$

while $a$ and $b$ are any arbitrary functions of $t$. Without loss of generality one can take $\lambda=0=\delta$. Thus the Noether operators for the system (4.1) will be:

$$
X_{1}=\frac{\partial}{\partial t}, \quad X_{2}=\frac{\partial}{\partial x}, \quad X_{3}=x \frac{\partial}{\partial V}, \quad X_{(a, b)}=a \frac{\partial}{\partial U}+b \frac{\partial}{\partial V} .
$$

Using (2.11) and after applying the inverse transformation, i.e. $U \rightarrow \int u d x, V \rightarrow \int v d x$ we can get the corresponding conserved vectors for the system (1.1). One can easily verify that only $X_{2}$ leads to local conserved quantity:

$$
T_{2}^{1}=-u v, \quad T_{2}^{2}=\frac{\Omega}{2} v^{2}-\frac{\Omega}{2} u_{x}^{2}+\frac{8 \Omega}{3} u^{3}+\Omega u u_{x x} .
$$

\subsection{Double reduction by Noether approach}

In this section, we will apply double reduction theory $[3,18,19]$ for the reduction of the system (1.1). One can see that $\mathbf{Y}_{\mathbf{1}}$ and $\mathbf{Y}_{\mathbf{2}}$ are the associated Lie point symmetries thus using a linear transformation:

$$
Y=\frac{\partial}{\partial t}+\beta \frac{\partial}{\partial x}
$$

the corresponding similarity variables will be:

$$
s=t, r=\beta t-x, u=u(r), v=v(r) .
$$

Using nonlocal conservation law (4.12) in (2.14) we have

$$
T^{r}=-\beta u v-\frac{\Omega v^{2}}{2}+\frac{\Omega u_{r}^{2}}{2}-\frac{8 \Omega u^{3}}{3}-\Omega u u_{r r} .
$$

Using Theorem (2.1) we get:

$$
-\Omega u \frac{d^{2} u}{d r^{2}}+\frac{\Omega}{2}\left(\frac{d u}{d r}\right)^{2}-\frac{8 \Omega u^{3}}{3}-\beta u v-\frac{\Omega v^{2}}{2}=K,
$$

where $K$ is a real constant.

So system (1.1) after reduction converts to:

$$
\frac{d v}{d r}=-\beta \Omega \frac{d u}{d r}, \quad-\Omega u \frac{d^{2} u}{d r^{2}}+\frac{\Omega}{2}\left(\frac{d u}{d r}\right)^{2}-\frac{8 \Omega u^{3}}{3}-\beta u v-\frac{\Omega v^{2}}{2}=K
$$




\subsection{Conclusion}

In this paper, we presented a complete classification of one dimensional subalgebra of the symmetry algebra of the system of shallow-water waves propagation up to conjugacy classes. These conjugacy classes were further used to reduce the considered system (1.1) into system of ordinary differential equation.

Further, Noether approach was applied to the non-variational third order systems of PDEs (1.1). In order to convert the considered system into variational problem the transformation $u=U_{x}$ and $v=V_{x}$ was applied. Moreover, local conserved quantity for new fourth order systems in $U, V$ variables was computed by using Noether approach. Then an inverse transformation $U \rightarrow \int u d x, V \rightarrow \int v d x$ was used to obtain the local conserved quantity for the main problem. The obtained local conservation law with corresponding Lie point symmetries was used to get double reduction for the system (1.1).

\section{References}

[1] M. Ablowitz and H. Segur, Solitons and the inverse scattering transform, SIAM Philadelphia, 1981.

[2] S. C. Anco and G. W. Blauman, Direct construction method for conservation laws of partial differential equation, Part II: General treatment, Euor. J. Appl. Math., 9 (2002), $567-585$.

[3] A. H. Bokhari, A. Y. Al-Dweika, F. D. Zaman, A. H. Kara and F. M. Mahomed, Generalization of the double reduction theory, Nonlinear Anal.: Real World Appl., 11(5) (2010), 3763 - 3769.

[4] A. F. Cheviakov, GeM software package for computaion of symmetries and conservation laws of differential equation, Comp. Phys. Commun., $176(2007), 48-61$.

[5] Yu. A. Chirkunov, E. O. Pikmullina, Symmetry properties and solutions of shallow water equations, Universal J. Appl. Math., 2 (1) (2014), 10 - 23.

[6] Ü. Göktas, W. Hereman, Symbolic computation of conserved densties for system of nonlinear evolution equation, J. Symb. Comput., 24 (1997), 591 621.

[7] W. Hereman, P. J. Adams, H. L. Eklund, M. S. Hickman, B. M. Herbst, Direct methods and symbolic software for conservation laws of nonlinear equations, In: Advances of Nonlinear Waves and Symbolic Computation, New york: Nova Science, Yan Z (Ed.), (2009), 19-79.

[8] W. Hereman, M. Colagrosso, R. Sayers, A. Ringler, B. Deconinck, M. Nivala et al., Continous and discrete homotopy operators and the computation of conservation laws, In: D. Wang, Z. Zheng (Ed.), Differential Equations with Symbolic Computation, Basel: Birkhäuser, (2005), 249 - 285.

[9] W. Hereman, Symbolic computation of conservation laws of nonlinear partial differential equations in multi-dimensions, Int. J. Quant. Chem., 106 (2006), $278-299$.

[10] A. H. Kara, F. M. Mahomed, Noether-type symmetries and conservation laws via partial Lagrangian, Nonlinear Dyn., 45 (2006), 367 - 383.

[11] F. Neyzi, M. B. Sheftel, D. Yazici, Symmetries, integrals and three dimensional reductions of Plebanski's second heavenly equation, Phys. Atom. Nuclei, $70(3)(2007), 584-592$.

[12] E. Noether, Invariante Variationsprobleme, Nacr. König. Gesell. Wissen., Göttingen, Math.-phys. Kl. Heft, 2 (1918), 235 - 257, (English translation in Transport Theo. Stat. Phy., 1(3) (1971), 186-207.

[13] P. J. Olver, Applications of Lie Groups to Differential Equations, Springer-Verlag, New York, 1986.

[14] M. Pandey, Exact solutions of the shallow water equations, Intl. J. Nonlinear Sci., 16 (2013), 334-339.

[15] A. D. Polyanin and V. F. Zaitsev, Handbook of Nonlinear Partial Differential Equation, CRC Press, Boca Raton London, 2004.

[16] J. Patera and P. Winternitz, Subalgebras of real three- and four-dimensional Lie algebras, J. Math. Phy., 18(7) (1977), $1449-1455$.

[17] T. R. Sekhar and B. Bira, Wave features and group analysis for axisymmetric flow of shallow water equations, Int. J. Nonlinear Sci., 14 (2012), 23 - 30.

[18] A. Sjöberg, Double reduction of PDEs from the association of symmetries with conservation laws with applications, Appl. Math. Comput., $84(2007), 608-616$.

[19] A. Sjöberg, On double reductions from symmetries and conservation laws, Non. Lin. Anal. Real World Appl., 10(6) (2009), 3472 - 3477.

[20] H. Steudel, Über die Zuordnung zwischen invarianzeigenschaften und Erhaltungssatzen. Z Naturforsch, 17A (1962), $29-32$.

[21] T. Wolf, A comparision of four approches to the calculation of conservation laws, Euorp. J. Appl. Math., 13 (2002), $129-152$.

[22] T. Wolf, A. Brand, M. Mohammadzadeh, Computer algebra algorithems and routines for the computations of conservation laws and fixing of guage in differential expressions, J. Symb. Comput., 27 (1999), $221-238$.

[23] D. Yazici, M. B. Sheftel, Symmetry reductions of second heavenly equations and 2+1 dimensional hamiltonian integrable system, J. Non. Lin. Math. Phys., 15(3) (2008), $417-425$. 